\title{
Modeling of Kidney Stones from Ultrasound Images based on Hybrid Regional Segmentation with Active Contours
}

\author{
Dominik Vilimek *, Jan Kubicek, Adela Kloudova, Alice Krestanova, Marek Penhaker, Martin \\ Cerny, Martin Augustynek, David Oczka and Daniel Barvik
}

VSB-Technical University of Ostrava, FEECS, K450, 17. Listopadu 15, Ostrava-Poruba, Czech Republic

\begin{abstract}
Kidney stones (nephrolithiasis) are among the most common kidney diseases. They are solid stones that arise from minerals dissolved in urine. The treatment of kidney stones depends primarily on the position, size and composition of the stones, as well as on the general health condition of the patient. However, early diagnosis is quite complicated. In this paper, we propose a fully automatic hybrid method for identification and features extraction of the kidney stones. Specifically, model is based on the multiregional segmentation and approximation of the kidney stone area. Methods consequently use concept of the active contours which are focused on extraction of the geometrical features. The method remarkably allowing for an objective monitoring and classification of the kidney stones. These results may play a role to overcome conventionally used clinical procedures where clinicians mark kidney stones manually, without software feedback.
\end{abstract}

Keywords: segmentation; kidney stones; ultrasound; active contours; regional segmentation

\section{Introduction}

Kidney stones are formed by the presence of a substance in the urinary tract. The kidney stones could arise either directly in the renal goblets or cause obstacle at the site of origin in the renal pelvis. From renal goblets they can spread further through the urinary tract and cause serious problems when leaving it. They are minerals mostly (small stones), which are very concentrated in the urine and tend to form crystals. It is a relatively common disease and the probability is approximately $10 \%$ for each person. Another complication of kidney stones is a $70 \%$ chance of recreating after successful treatment. For these reasons, early and accurate diagnosis is crucial [1, 2].

The exact cause of kidney stones is not fully understood, but it is assumed that the risk factors affecting the disease are inadequate drinking regime, unhealthy diet, frequent urinary track disease, some types of drugs and heredity [3]. Imaging methods like ultrasound and CT are most often used to prove the presence of calculi. On ultrasonic examination, calculi are sometimes very difficult to recognize, but if it is localized we can measure their size. If the stone gets into the ureter, it is almost impossible to detect it by ultrasound, which is one of the main disadvantages. Primarily, ultrasound examination is used for diagnosis, because it does not burden the body, on the other hand X-ray is used for detect calcium kidney stones [4].

\section{Related Work}

The basis for ultrasonic image processing is preprocessing, which can enhance 
image contrast. In most cases, speckle noise often occurs in these images. To eliminate noise, it is suggested to use some filters i.e. a Gabor filter and then use histogram equalization to increase the contrast [5].

Another way to remove speckle noise from ultrasonic images is to use the despeckling wavelet thresholding. Speckle noise significantly affects the detection of lesions in the image and making image worse readable. They are two ways of using wavelet thresholding so called soft thresholding and hard thresholding. Comparison of these two methods shows, that more convenient could be soft thresholding $[6,7]$.

In different approach, it can be used level set segmentation and artifical neural network (ANN) classification for segmentation kidney stone from images, which were taken by ultrasound device. In the first step is important preprocessing which consists from reducing speckle noise for example using some filters like Gabor filter and use of contrast enhancement. In the second step is applied level set segmentation based on Symlets, Biorthogonal, Daubechies wavelet. In the last step is applied ANN classification for identifying kidney stones [8].

Another offered option is the noise removal based on the median filter. Since the kidney stones have a higher contrast than the surrounding structures, the segmentation algorithm is divided into two phases. In the first phase, high value objects are identified as potential kidney stones and in the second step, these objects are assessed to determine whether there is a shadow in the objects, they are designated as a kidney stone $[9,10]$.

For segmentation kidney stone from 3D abdominal ultrasound images, the team of Marsousi et al. developed algorithm based on shape model. In preprocessing, the noise of 3D ultrasound images was reduced by Gausian-Hamming filter and histogram equalization. Detection algorithm was searching for kidney shape in binarized volume. Probabilistic kidney shape model (PKSM) was using as initiation in segmentation, so boundaries of actual kidneys were closer [11].
As was mentioned above CT scans can also be used to diagnose kidney stones. When segmentation of the image allows identification of kidney stone. The method is based on 3 steps such as image preprocessing, level set segmentation and the analysis of kidney stones, if identified. Some automatic methods could be found in work of authors $[12,13]$.

\section{Proposal of Algorithm for Kidney Stones Segmentation from Ultrasound Images}

The proposed segmentation method is based on multithresholding and active contours. Method was tested on 30 ultrasound images divided into two datasets, which were taken in urological department in University Hospital Opava, Czech Republic. Figure 1 shows block diagram of proposed algorithm.

The histogram could be used to show the intensities contained in the image. With histogram equalization it's possible to achieve optimum contrast results. Moreover, it is available to do this on the whole image or in individual parts of the image.

\subsection{Preprocessing of image}

In the first step was necessary preprocess ultrasound images with artefacts such as motion artefacts, noise etc. Due preprocessing, the images were in better quality of contrast. The ultrasound images are mainly corrupted by speckle noise. After reading the image itself, the $\mathrm{ROI}$ was extracted, an area of interest in which kidney stones were located. Next step was converting images from RGB to grayscale and applied convolution for filtering the images. After removing the noise, the median filter was used for smoothing images. The mainly disadvantages of this method was losing some images details, so we applied Wiener's filter for preserve these details. In next subsection we made comparison of both filters.

\subsection{Preprocessing of image}

In this part we evaluated tested filters median and Wiener filter based on parameter Brisque and Niqe. These parameters will be explained below. As you can see in fig. 2 and fig 3., there are ultrasound

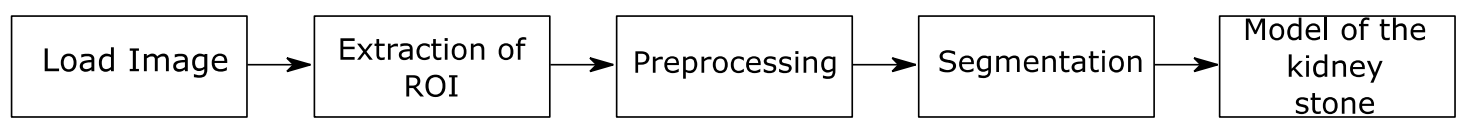

Figure 1: Proposed algorithm for modeling kidney stones from ultrasound images. 

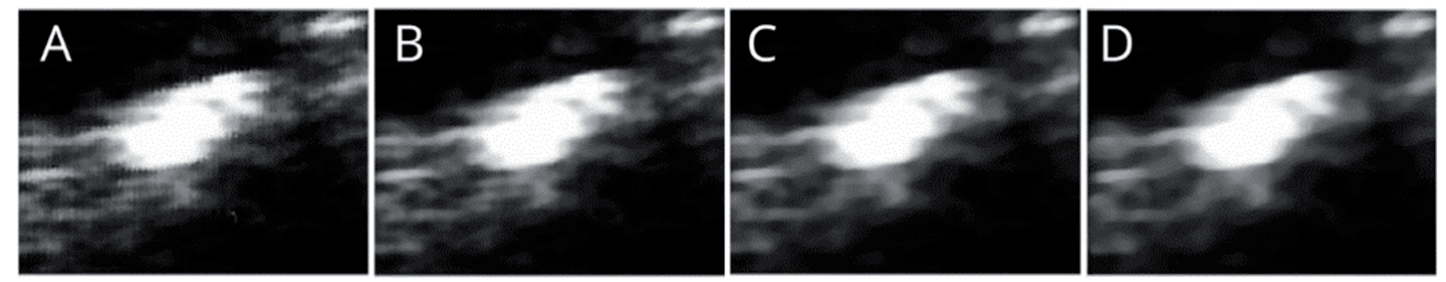

Figure 2: A native image with $\mathrm{ROI}$ - kidney stone, B image after median filtration with size of mask $6 \times 6, \mathrm{C}$ image after median filtration with size of mask12x12, D image after median filtration with size of mask 15x15.
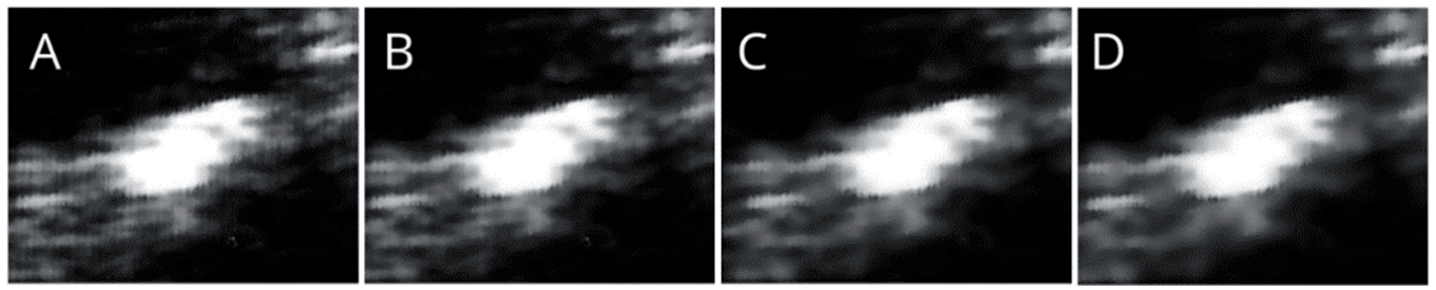

Figure 3: A native image with ROI - kidney stone, B image after Wiener filtration with size of mask 6x6, C image after Wiener filtration size of mask12x12, D image after Wiener filtration with size of mask $15 \times 15$.

image after application median and Wiener filter with different size of mask. The quality of images was quantitatively tested based on two parameters Brisque and Niqe. These no-reference methods testing images based on fact, that reference image does not exist. Parameter Brisque evaluate quality of image against calculated model from images with similar distortion as evaluated image. The lower the score is, the better quality image has. Parameter works with calculation statistics in image scene with local normalized coefficients of brightness for quantify losses of naturalness of image, which comes from presence of distortion. So, this way measure of quality could be evaluated holistically $[14,15]$.

The fig. 4 below show results with Brisque evaluation of median and Wiener filter.

Figures 2 and 3 compare the differences in application of the median and Weiner filter for ultrasound image smoothing. Based on values of Brisque parameter, which are shown at fig. 4 it can be assumed that better result was acquired for the first datataset by use of median filter. On the other hand, for the second dataset was more reliable Wiener's filter. It could be caused by different bad quality of images, which are worse in the dataset. When we take advantage the proportion between individual filters in Figure 4, we can note more significant differences in the dataset 2 , whereas more reliable the Weiner's filter appears.
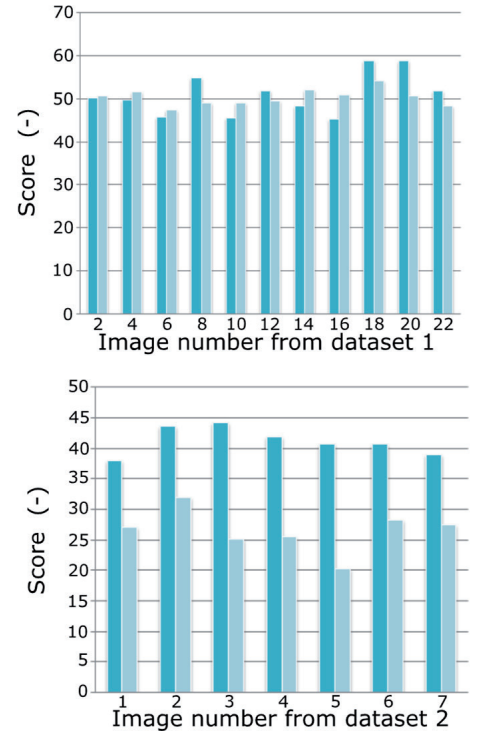

Figure 4: Brisque parameters, where darker blue represents the Median filter and lighter blue represents the Wiener filter.

Parameter of Naturalness Image Quality Evaluator (NIQE) calculating score against default value of image. The smaller score means better image quality. The Niqe parameter is based on the Brisque parameter calculation but use only the Natural Scene Statistics (NSS) of the image properties. The main advantage of NSS method is ability to be not tied to any type of distortion. Image is divided into PXP patches. Using the NSS, from 
patches with size PxP from analysed image. Quality of image is comparing with Multi-Variate Gaussian model (MVG). Quality of the distorted image is classified as distance between quality model NSS and MVG model by equation [7]:

$$
D\left(v_{1}, v_{2}\right)=\sqrt{\left(v_{1}-v_{2}\right)^{T} \cdot\left(\frac{\sum_{1}+\sum_{2}}{2}\right)^{-1} \cdot\left(v_{1}-v_{2}\right)}
$$

where $v_{1}, v_{2}$ are mean vectors and $\Sigma_{1}+\Sigma_{2}$ are covariance matrices of the natural MVG model and the distorted image's MVG model [7, 16]. The fig 5 below show results with NIQE evaluation of median and Wiener filter.

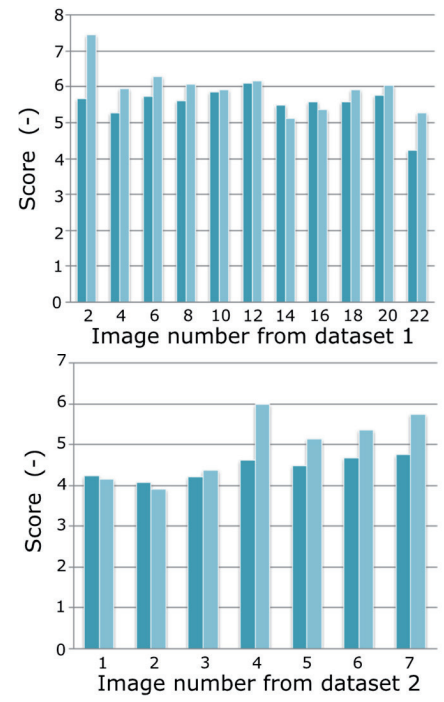

Figure 5: NIQE parameters, where darker blue represents the Median filter and lighter blue represents the Wiener filter.
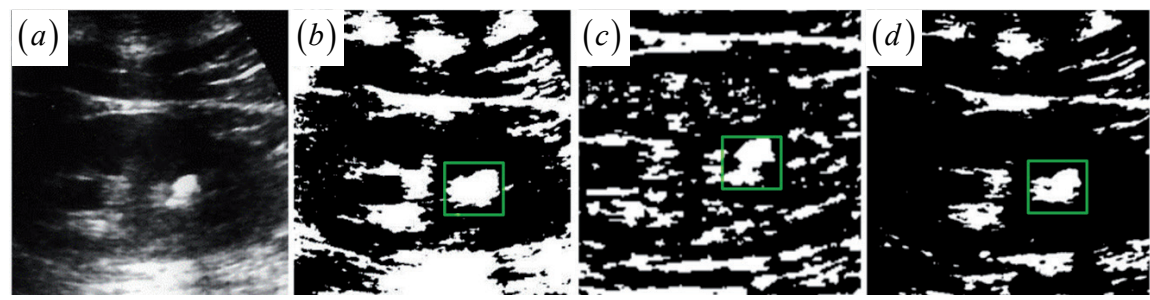

Figure 6: a) Native image, b) Threshold value 0.5 , c) Threshold value 0.75 , d) Threshold value 1.
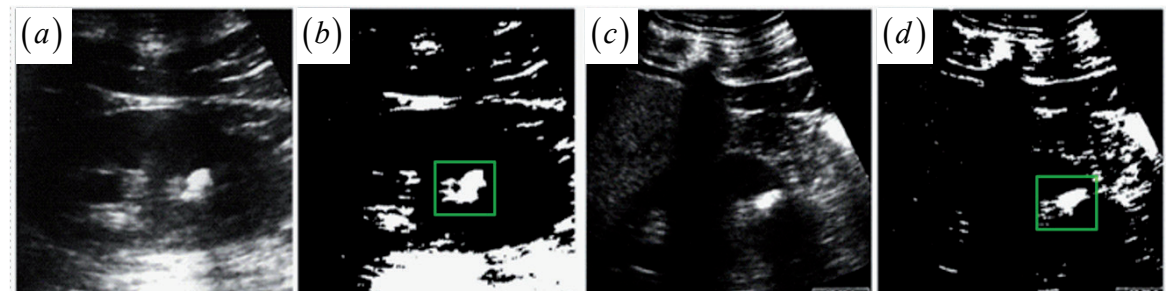

Figure 7: a)Tested native image, b) Image after calculation of threshold, c) Tested native image from different dataset, d) Image after calculation of threshold.
From the results of Brisque and NIQE parameters could be concluded that the median filter appears better for image smoothing than the Wiener filter. For this reason, a median filter was used for preprocessing.

\subsection{Segmentation of kidney stones}

For segmentation of kidney stones we tested image binarization, automatic calculating threshold by interclass variance, manual threshold selection and Otsu thresholding. The optimal method for segmentation of kidney stones was searched.

Firstly, we tested simply segmentation operation called binarization. We set the thresholds values $0.5,0.75,1$. Pixels which were under the threshold, got value 0 and pixels above the threshold got value 1 . In another words 0 for black colour and 1 for white colour. Output of binarization was black and white image. The results are shown in the fig. 6. As expected, the binarization was not suitable for segmentation algorithm, because the method detects a lot of parts in image, which were not part of the kidney stone. For a clearer view the kidney stone can be find in the green rectangle.

Secondly, we tested method with calculation of threshold based on minimizing the intraclass variance. Fig. 7 shows kidney stones in the green rectangle. This method looked insufficient as well. There were many segmented parts that misrepresented kidney stone.

Thirdly, we made experiment with manual selected threshold. Even simpler method for segmentation is to distinguish the objects from the 

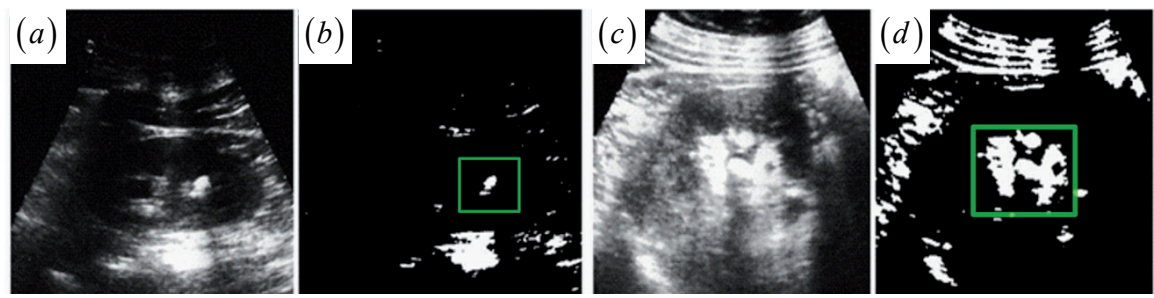

Figure 8: a)Tested native image, b) Segmented images with manual setting threshold value 220, c) Tested native image from different dataset, d) Segmented images with manual setting threshold value 220.

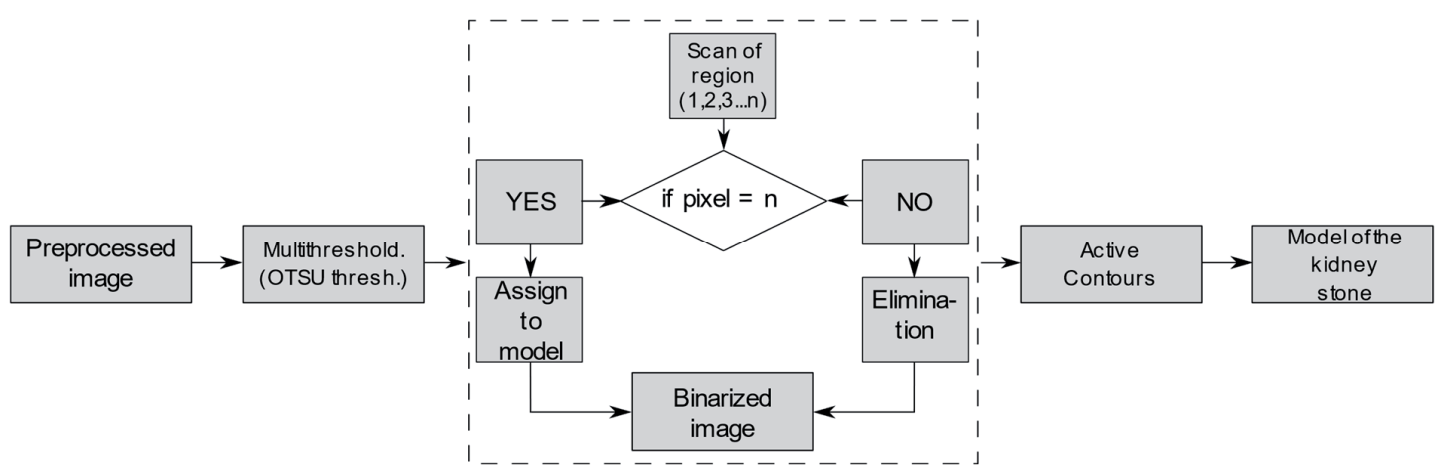

Figure 9: Proposed algorithm for segmentation kidney stones.

background based on the tint of each pixel. Only a one threshold value was set which separates pixels that represent black or white. Manually we set threshold value on 220. Again, the pixels under this threshold were set to black (value 0 ) and above the threshold to white (value 255). The results seem to be good see fig. 8 , because in the image wasn't lot of different parts. However, the problem comes with manual selection of threshold, so for this reason method is not suitable.

Finally, we were testing Otsu thresholding, which is type of multiregional segmentation. This method is depended on the principle of finding the optimal threshold for image segmentation. The threshold is obtained based on the intra or inter-scattering of individual pixels. In other words, decision which pixel will be eliminated and which will not. Based on the Otsu thresholding, the entire image is divided into the appropriate number of regions according to the ideally selected thresholds. The result was a binarized image where only the class containing the desired object was extracted. The whole proposed algorithm for segmentation is shown at fig. 9.

The fig. 10 shows implementation of algorithm for ultrasound images.

Different set of threshold values is show on fig. 11. Specifically, values were set 2, 3, 4, 5, 6 thresholds. The fig. 11 shows, that optimal setting for segmentation of kidney stones is 5 threshold value with 6 regions. When was set less thresholds, the segmentation of kidney stone was insufficient.
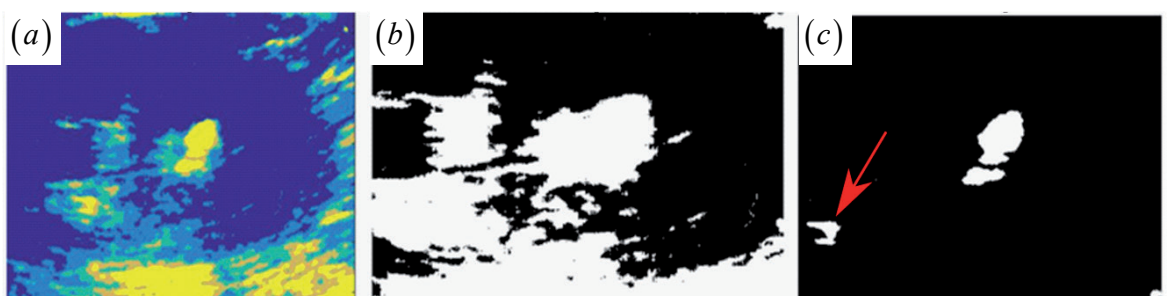

Figure 10: Implementation of algorithm for multiregional segmentation: a) Image divide into 5 regions, b) Extraction of ROI, c) Extraction of region with object of interest, Red arrow show structure of expected kidney stone. 

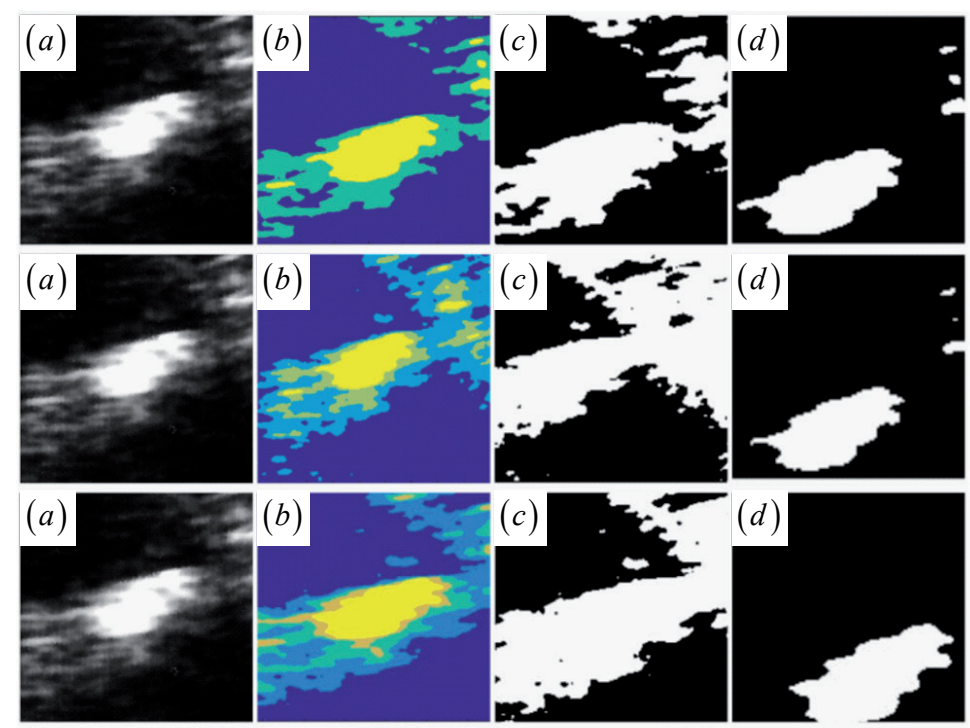

$(d)$
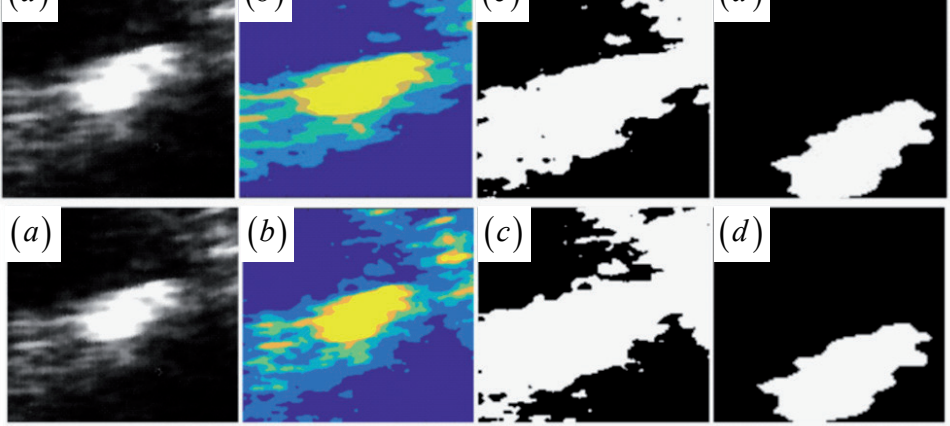

Figure 11:a) Native image, b) Multiregional segmentation with 2, 3, 4, 5 thresholds (from above), c) extraction by ROI, d) Extraction of kidney stone.

\subsection{Algorithm for modelling kidney stones morphology}

For modelling kidney stones was used method so called active contours. Mathematically active contour could be described by equation below:

$$
\begin{aligned}
E_{\text {snake }}^{*}(v) & =\int_{0}^{1} E_{\text {snake }}(v(s)) d s=\int_{0}^{1} E_{\text {int }}(v(s))+E_{\text {ext }}(v(s)) d s= \\
& =\int_{0}^{1} E_{\text {int }}(v(s))+E_{\text {image }}(v(s))+E_{\text {con }}(v(s)) d s
\end{aligned}
$$

where $E_{\text {int }}$ is internal energy, $E_{\text {ext }}$ is external energy, $E_{\text {image }}$ means energy calculated from image and $E_{\text {con }}$ represent points of initialization curve.

Principally the method use initialization curve placed on object. Segmentation is based on internal and external energy of the image. In the output image, the kidney stone is bordered by a red line. Active contours are dependent on the number of iterations, the higher number of iterations is, the object will be better bordered. However, the computational complexity will be higher. As you can see below, set number of iterations was better with value 900 than 100. It can be assumed if we set smaller value than 100 iterations, the modelling of kidney stone will be inadequate.
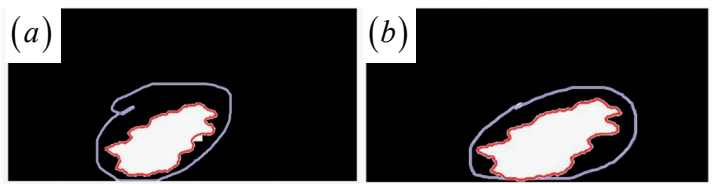

Figure 12: a) Active contour model with the initial contour as a rough approximation of ellipse and final segmentation result, indicated red. Active contour with number of iterations 100, b) Active contour with number of iterations 900 .

\section{Conclusions}

In this paper, dataset with 30 ultrasound images was tested. For preprocessing was tested median filter and Wiener filter. Quality evaluation was based on parameters Brisque and Niqe. According to results the median filter was more suitable for image preprocess.

For segmentation algorithm we tested various methods. Firstly, the simplest method of segmentation, binarization. This method was not sufficient for further processing although we tried to set different threshold levels, the kidney stone could not be sufficiently separated from the surrounding structures. Even by calculating the threshold for each frame separately, we did not succeed. Therefore, we 
decided to use multiregional segmentation, which divided the whole image based on the scattering of luminance values into several regions. Based on experimental measurements we founded out that the ideal number of regions was 6 regions and therefore 5 threshold values were used.

Using the initialization curve I limited only the kidney stone structures, which were subsequently bounded by a red curve. Based on the extraction of parameters from the kidney stone model, it was possible to calculate the area, perimeter and length of the main and minor half-axes. Area calculation was calculated in two ways, the first was the counting of white pixels after multiregional segmentation and the second was the calculation of pixels that were inside the active contour.

This work also had its limitations, which could be the subject of further work. The first was a relatively small test dataset, which was used mainly to test the most appropriate segmentation. Other methods such as the marching level set could also be tested. Future work would lead to the creation of a software application that would compute all kidney stone parameters of interest on this principle.

\section{Acknowledgments}

The work and the contributions were supported by the project SV450994/2101 Biomedical Engineering Systems XV'. This study was also sup-ported by the research project The Czech Science Foundation (GACR) 2017 No. 17-030375 Investment evaluation of medical device development run at the Faculty of Informatics and Management, University of Hradec Kralove, Czech Republic. This study was supported by the research project The Czech Science Foundation (TACR) ETA No. TL01000302 Medical Devices development as an effective investment for public and private entities.

\section{References and Notes}

[1] Geraghty, R.M., Jones, P., Somani, B.K.: Worldwide Trends of Urinary Stone Disease Treatment Over the Last Two Decades: A Systematic Review. Journal of Endourology 31 (6), 547-556 (Jun 2017)

[2] Khan, S.R., Pearle, M.S., Robertson, W.G., Gambaro, G., Canales, B.K., Doizi, S., Traxer, O., Tiselius, H.G.: Kidney stones. Nature Reviews Disease Primers 2(1), 16008 (Dec 2016)

[3] Rocca Rossetti, S.: Kidney Stones. In: Managing Segmental Renal Diseases, pp. 15\{18. Springer International Publishing, Cham (2018)

[4] Roberson, N.P., Dillman, J.R., O'Hara, S.M., DeFoor, W.R., Reddy, P.P., Giordano, R.M., Trout, A.T.: Comparison of ultrasound versus computed tomography for the detection of kidney stones in the pediatric population: A clinical efectiveness study. Pediatric Radiology 48(7), $962\{972$ (Jul 2018)

[5] Viswanath, K., Gunasundari, R., Hussain, S.A.: Analysis of Kidney stone Detection by Reaction diffusion Level Set Segmentation and Xilinx System Generator. In: Proceedings of the 2015 International Conference on Advanced Research in Computer Science Engineering \& Technology (ICARCSET 2015) - ICARCSET '15. pp. 1 \{9. ACM Press, Unnao, India (2015)

[6] Akkasaligar, P.T., Biradar, S.: Diagnosis of renal calculus disease in medical ultrasound images. In: 2016 IEEE International Conference on Computational Intelligence and Computing Research (ICCIC). pp. 1\{5. IEEE, Chennai (Dec 2016)

[7] Goel, N., Yadav, A., Singh, B.M.: Medical image processing: A review. In: 2016 Second International Innovative Applications of Computational Intelligence on Power, Energy and Controls with Their Impact on Humanity (CIPECH). pp. 57 \{62. IEEE, Ghaziabad, India (Nov 2016)

[8] Viswanath, K., Gunasundari, R.: Design and analysis performance of kidney stone detection from ultrasound image by level set segmentation and ANN classi_cation. In: 2014 International Conference on Advances in Computing, Communications and Informatics (ICACCI). pp. 407\{414. IEEE, Delhi, India (Sep 2014)

[9] Akkasaligar, P.T., Biradar, S., Badiger, S.: Segmentation of Kidney Stones in Medical Ultrasound Images. In: Santosh, K.C., Hegadi, R.S. (eds.) Recent Trends in Image Processing and Pattern Recognition, vol. 1036, pp. 200\{208. Springer Singapore, Singapore (2019)

[10] Vijayakumar, M., Ganpule, A., Singh, A., Sabnis, R., Desai, M.: Review of techniques for ultrasonic determination of kidney stone size. Research and Reports inUrology Volume 10, $57\{61$ (Aug 2018)

[11] Marsousi, M., Plataniotis, K.N., Stergiopoulos, S.: Shapebased kidney detection and segmentation in threedimensional abdominal ultrasound images. In: 2014 36th Annual International Conference of the IEEE Engineering in Medicine and Biology Society. pp. 2890\{2894\}. IEEE, Chicago, IL (Aug 2014)

[12] Akkasaligar, P.T., Biradar, S., Kumbar, V.: Kidney stone detection in computed tomography images. In: 2017 International Conference On Smart Technologies For Smart Nation (SmartTechCon). pp. 353\{356. IEEE, Bangalore (Aug 2017)

[13] Guanyu Yang, Jinjin Gu, Yang Chen, Wangyan Liu, Lijun Tang, Huazhong Shu, Toumoulin, C.: Automatic kidney segmentation in CT images based on multi-atlas image registration. In: 2014 36th Annual International Conference 
of the IEEE Engineering

[14] Mittal, A., Moorthy, A.K., Bovik, A.C.: No-Reference Image Quality Assessment in the Spatial Domain. IEEE Transactions on Image Processing 21(12), 4695\{4708\}. (Dec 2012)

[15] Outtas, M., Zhang, L., Deforges, O., Hamidouche, W., Serir, A.: Evaluation of No-reference quality metrics for Ultrasound liver images. In: 2018 Tenth International Conference on Quality of Multimedia Experience (QoMEX). pp. 1\{3. IEEE\}, Cagliari (May 2018)

[16] Mittal, A., Soundararajan, R., Bovik, A.C.: Making a । Completely Blind" Image Quality Analyzer. IEEE Signal Processing Letters 20(3), 209\{212 (Mar 2013) 